\title{
Instantaneous Electromagnetic Torque Waveform Calculations for Switched Reluctance Machines Exploiting Vector Analysis
}

\author{
Aleksas Stuikys ${ }^{1}$, Bahareh Zaghari $^{2}$, Member, IEEE, and Jan K. Sykulski ${ }^{2}$, Fellow, IEEE \\ ${ }^{1}$ RETORQ Motors Ltd., London, UK \\ ${ }^{2}$ School of Electronics and Computer Science, University of Southampton, Southampton, SO17 1BJ, UK
}

\begin{abstract}
Switched Reluctance (SR) machines are magnetically non-linear electromechanical devices and their full and accurate performance prediction - especially of the instantaneous electromagnetic torque waveforms - is not amenable to closed form analytical solutions. Consequently, the analysis of such machines is usually performed with the aid of computer based numerical simulations that - even if providing good accuracy - do not offer physical insight into the electromagnetic energy conversion processes taking place inside the machine. For this reason, the SR machine design and analysis are not simple exercises as they require substantial computational resources and extensive prior design expertise. In this paper, a methodology for a reasonably accurate prediction of the instantaneous electromagnetic torque waveforms of the SR machines is proposed using a closed form analytical solution. The suggested approach relies on a simple vector analysis of the flux-linkage map of a non-linear SR machine and as such avoids integration, non-linear curve fitting or geometrical series summation. The proposed vector analysis based methodology offers intuitive physical insight into the electromagnetic energy conversion processes taking place inside the SR machine related to the instantaneous torque generation.
\end{abstract}

Index Terms - Instantaneous electromagnetic torque, switched reluctance machines, tractive electromagnets, vector analysis.

\section{INTRODUCTION}

$\mathrm{A}^{\mathrm{s}}$ SWITCHED RELUCTANCE (SR) MACHINE is a type of an electromechanical device used to convert electrical into mechanical energy when operated in the motoring mode, or mechanical into electrical energy when in the generating mode [1]. SR machines can be constructed as rotating or translating devices, depending on particular application requirements [2].

Since SR machines are expected to work economically, it is very helpful to be able to design and predict their performance accurately using simple and intuitive analytical methods at an early design stage to minimize engineering development costs. Such methods should provide insight into the operating principles and electromagnetic energy conversion processes taking place inside the machines. Among the most important quantities to be predicted in the SR machine design process are the rated instantaneous and average electromagnetic torque. An accurate prediction of the torque enables the designer to size effectively the machine for a particular power requirement. However, since the SR machines are magnetically non-linear, the instantaneous torque waveform prediction is generally not possible as an analytically closed form solution [3].

The earliest attempt to predict the non-linear SR machine instantaneous electromagnetic torque waveforms using closed form analytical expressions, avoiding analytical or numerical integration, was made in [4], where it was argued that although the machines were generally magnetically non-linear their characteristic flux-linkage map could be split into three distinct regions. Two of these regions are non-linear and can be modelled using the Fröhlich-type equations, whereas the

Manuscript received December 2019; revised June 2020; accepted October 2020. Date of publication; date of current version. Corresponding author: A. Stuikys (e-mail: a.stuikys.ac@gmail.com).

Color versions of one or more of the figures in this paper are available online at http://ieeexplore.ieee.org.

Digital Object Identifier (inserted by IEEE). third region is amenable to linearization and can thus be modelled using straight-line equations to describe the relationship between the rotor angle and the flux-linkage. Therefore, the method presented in [4] is of the analytically closed form requiring no integral solutions and can be easily parametrized in terms of the phase current and the rotor angle. The comparison of the computer generated instantaneous torque waveforms with the analytical method indicated relatively close agreement [4]. However, due to the discontinuous treatment of the three regions of the fluxlinkage map, the analytical method in [4] was not in a convenient form to offer simplicity, intuition and insights into the energy conversion processes, as numerous curve fitting coefficients were necessary in order to evaluate the three separate equations over the entire range of rotor angles.

An alternative analytical approach to predict the electromagnetic torque waveforms of saturable SR machines was proposed in [5]. The non-linear flux-linkage map of the SR machine was represented by a Fourier series, recognizing the periodic nature of the flux-linkage function with respect to the rotor angle and fitting the optimized non-linear polynomial equations. The Fourier series based flux-linkage representation enables the summation of the geometric series, thus obtaining the instantaneous torque waveform with the phase current and rotor position as parameters. The comparison between the results obtained using the analytical approach with the magnetic field Finite Element Analysis (FEA) simulations showed relatively good agreement. The approach in [5] avoids the need for direct analytical integration in the computation of the torque; however, it falls short of the mathematical closedform solution in that the resulting Fourier series summation expression does not offer the simplicity and insights into the energy conversion processes because of the numerous curve fitting coefficients, otherwise having no physical meaning, used in the expressions.

A more recent attempt to express the instantaneous 
electromagnetic torque of saturable SR machines using a closed form mathematical solution was presented in [6]. The non-linear flux-linkage map of the saturable SR machine was modelled with the analytical arctangent function, thus expressing the phase-current/flux-linkage relationship analytically. The flux-linkage function with respect to the rotor position of the machine was modelled using a general Fourier series summation approach. The resulting instantaneous electromagnetic torque expression was derived in terms of two relationships of the flux-linkage and integrated analytically [6]. This resulted in the instantaneous torque expressed in terms of the phase current and the rotor position. Such an instantaneous torque expression offers the advantage of being parametrized, although requires numerous curve fitting coefficients - and non-intuitive relationships between these coefficients - rendering the expression useful only for computer aided SR machine dynamic operation calculations; moreover, it offers no physical insights into the electromagnetic energy conversion processes inside the SR machines.

The foregoing overview points to the following shortcomings of the existing methods: a) the need for analytical or numerical integration, b) the requirement for nonlinear curve fitting with the associated fitting coefficients, and c) the resulting expressions for the instantaneous torque function offering no or little physical insight. As the understanding of the electromechanical energy conversion processes is not helped, it becomes difficult to perform the initial machine design calculations without resorting to highly sophisticated and specialized computer software and/or profound expertise in the design of such machines. What is needed at the preliminary stages of the SR machine electromagnetic circuit design is an analytically simple expression of the instantaneous electromagnetic torque with respect to phase current and rotor position that does not require integration and does not depend on non-linear curve fitting techniques or Fourier series based summations.

In this paper, we set out to derive a computationally simple procedure to compute instantaneous electromagnetic torque waveforms of saturable SR machines based on vector analysis. The proposed analytical expression is intuitive, avoids integration and does not require non-linear curve fitting or geometric series summations.

\section{STATIC ELECTROMAGNETIC TORQUE WAVEFORM COMPUTATIONS}

\section{A. Electromagnetic Energy Conversion Principles}

Since SR machines are magnetically non-linear, their fluxlinkage map has to be represented as a function of both the phase current and the rotor position, as shown in Fig. 1 for a particular 18/12 SR machine topology (indicating 18 stator poles and 12 rotor poles). The flux-linkage curve for each rotor position is unique as the magnetic non-linearity is highly pronounced at the fully aligned rotor position - coinciding with the saturation phase current $i_{s}$ - but saturation being almost absent in the fully unaligned rotor position and beyond the rated phase current $i_{r}$. The flux-linkage map shown in Fig. 1 is for the half of the rotor pole pitch, which for the 18/12 SR machine is $30^{\circ}$. It is worth noting that although the results in Fig. 1 were generated using 2D FEA software [7], yielding quite accurate results, similar accuracy is achievable by using the flux-tubes magnetic analysis technique proposed in [8], with significant savings in computational effort and time. Alternatively, analytical methods proposed in [9] and [10] could be used to obtain the fully non-linear flux-linkage curves as in Fig. 1, since these methods do not require any magnetic field FEA or measured magnetisation data.

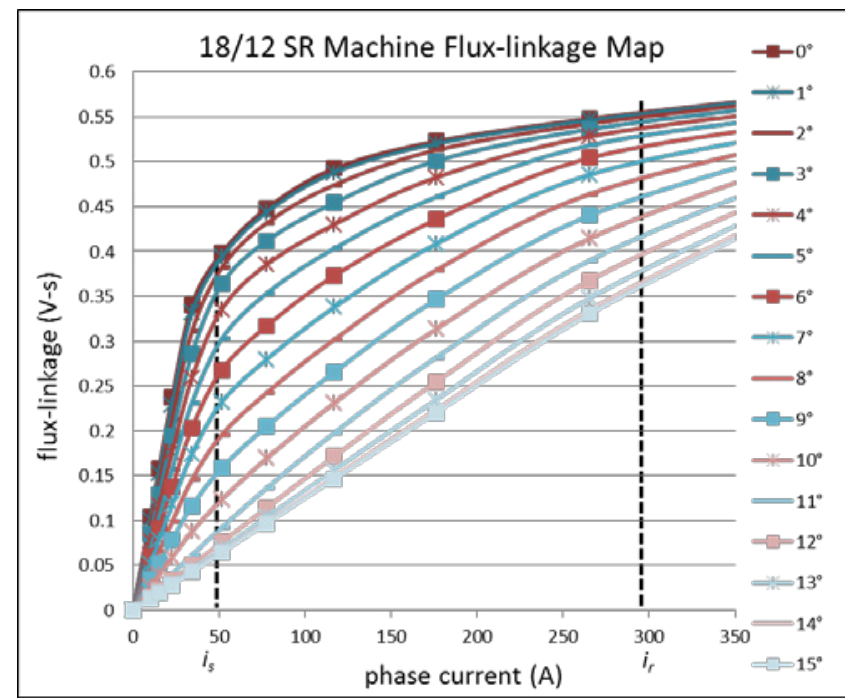

Fig. 1. A non-linear flux-linkage map with the indicated saturation and rated phase currents $\left(\boldsymbol{i}_{\boldsymbol{s}}, \boldsymbol{i}_{\mathbf{r}}\right)$ for an 18/12 topology SR machine.

The instantaneous electromagnetic torque of the SR machines may be found by differentiating the co-energy with respect to the rotor position at a given phase current as [1]

$$
T=\left[\frac{\partial W^{\prime}}{\partial \theta}\right]_{i=\text { constant }}
$$

where the co-energy $W^{\prime}$ represents the area bounded by the fully aligned and the fully unaligned flux-linkage curves of the flux-linkage map in Fig. 1 and $i$ is the instantaneous phase current value corresponding to a particular rotor angle. Clearly, expressing the generally non-linear flux-linkage curves in terms of known mathematical functions will result in an exact analytical expression for $W^{\prime}$, which - when subsequently differentiated in (1) - can be parametrized in terms of the phase current and the rotor position to yield the instantaneous electromagnetic torque waveform at each rotor angle. However, as noted earlier, a mathematically simple representation of the flux-linkage function in terms of the phase current and the rotor angle is seldom possible. Notwithstanding, recognizing that it is possible to represent relatively accurately the non-linear flux-linkage map of Fig. 1 using linearization techniques, together with Varignon's parallelogram [11], for the estimation of the SR machine coenergy $W^{\prime}$ [12], it then becomes a simple matter to obtain the rated average electromagnetic torque of the SR machine as 
shown schematically in Fig. 2.

The flux-linkage linearization technique is relatively simple, requiring - at most - two linear curves to represent the non-linear flux-linkage corresponding to each rotor position, with Fig. 2 showing an example for the fully aligned and fully unaligned rotor positions. The respective linearized inductances $L$ may be found by the ratio of the difference of respective flux-linkages to the difference of respective phase currents [1]. The co-energy area $W^{\prime}$ ' (in this work expressed in units of joule) of the general quadrilateral $V_{0} V_{1} V_{2} V_{3} V_{0}$ formed so by the phase current trajectory vectors $V$ in Fig. 2 can, with application of Varignon's quadrilateral, be found as (the full derivation details are given in [12])

$$
\begin{gathered}
W^{\prime}=\frac{1}{2}\left|\left(\left(V_{2}-V_{0}\right) \times\left(V_{3}-V_{1}\right)\right)\right|= \\
=\frac{1}{2}\left[\left(L_{s a}-L_{u u}\right) i_{r}{ }^{2}+\left(L_{u a}-L_{s a}\right) i_{s} i_{r}+\Psi_{s} i_{r}-\Psi_{s} i_{s}\right]
\end{gathered}
$$

where $L_{s a}$ is the saturated aligned inductance, $L_{u u}$ is the unsaturated unaligned inductance, $L_{u a}$ is the unsaturated aligned inductance, $\Psi_{s}$ is the flux-linkage axis intercept, $i_{r}$ is the rated phase current and $i_{s}$ is the saturated phase current.

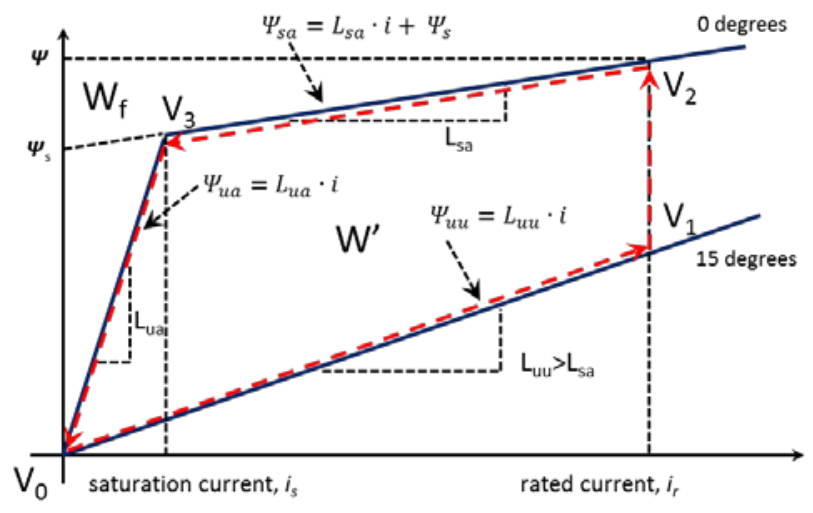

Fig. 2. Linearized flux-linkage curves for the generally non-linear fluxlinkage curves of Fig. 1. In the figure only the fully aligned and the fully unaligned linearized flux-linkage curves are shown.

Having estimated the maximum value of the electromagnetic energy conversion area for a given SR machine using (2), the average rated electromagnetic torque for the SR machine can then be computed using

$$
T=W^{\prime} \times \frac{q \cdot N_{r}}{2 \pi}
$$

where $q$ is the number of phases and $N_{r}$ is the number of rotor poles [1].

Equation (2) can be put to further use, this time for the instantaneous electromagnetic torque computations at each rotor angle, from the fully aligned to the fully unaligned positions, in selected increments of the rotor angle, recognizing that each successive linearized non-linear fluxlinkage curve together with the linear fully unaligned fluxlinkage curve forms a unique quadrilateral of its own. Examples of two such overlapping quadrilaterals are shown in
Fig. 3.

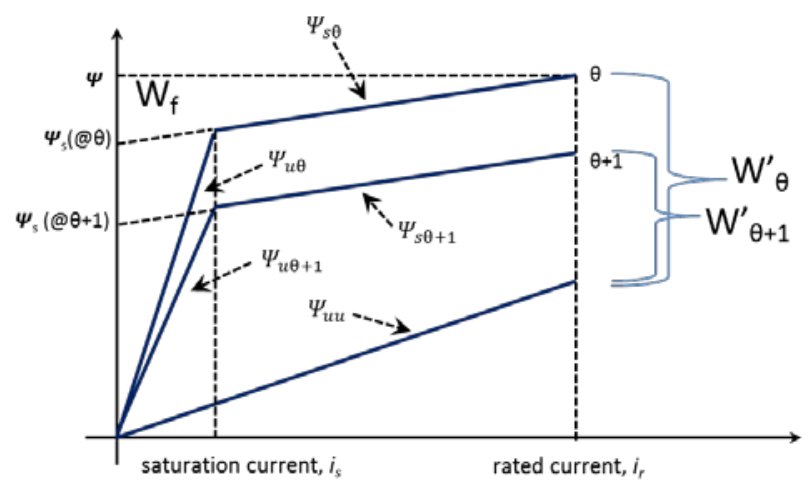

Fig. 3. Two overlapping quadrilaterals $W_{\theta}$ ' and $W^{\prime}{ }_{\theta+1}$ of the two linearized non-linear flux-linkage functions.

In Fig. 3 both of the quadrilaterals have their own areas $W$, easily computable with (2). Furthermore, it can be deduced from Fig. 3 that - since $W^{\prime}{ }_{\theta}$ represents a larger area than $W^{\prime}{ }_{\theta+1}$ - their difference, in turn, will represent an incremental change in the co-energy with respect to the incremental change in the rotor position as it travels from $\theta \rightarrow \theta+1$. Putting the last statement into a mathematical form by expressing each co-energy using (2) results in the incremental co-energy difference being given by

$$
\begin{gathered}
\Delta W^{\prime}=W_{\theta}^{\prime}-W_{\theta+1}^{\prime}=\frac{1}{2}\left[\left(L_{s \theta}-L_{u u}\right) i_{r}{ }^{2}+\left(L_{u \theta}-\right.\right. \\
\left.\left.L_{s \theta}\right) i_{s} i_{r}+\Psi_{s}(@ \theta) i_{r}-\Psi_{s}(@ \theta) i_{s}\right]-\frac{1}{2}\left[\left(L_{s \theta+1}-L_{u u}\right) i_{r}{ }^{2}+\right. \\
\left.\left(L_{u \theta+1}-L_{s \theta+1}\right) i_{s} i_{r}+\Psi_{s}(@ \theta+1) i_{r}-\Psi_{s}(@ \theta+1) i_{s}\right]= \\
=\frac{1}{2}\left[\Delta L_{s}\left(i_{r}{ }^{2}-i_{s} i_{r}\right)+\Delta L_{u} i_{s} i_{r}+\Delta \Psi_{s}\left(i_{r}-i_{s}\right)\right]
\end{gathered}
$$

where, on the right hand side of (4), $\Delta L_{s}=L_{s \theta}-L_{s \theta+1}$, $\Delta L_{u}=L_{u \theta}-L_{u \theta+1}$ and $\Delta \Psi_{s}=\Psi_{s}(@ \theta)-\Psi_{s}(@ \theta+1)$ as per Figs. 2 and 3. The right hand side of (4) is especially simple to interpret being composed of the incremental differences of the respective inductances and the respective flux-linkage axis intercepts, also noting that the unsaturated unaligned inductance $L_{u u}$ from Fig. 2 is not featuring at all in (4) due to the cancellation.

Having represented the incremental co-energy difference in terms of the incremental rotor angle change in (4) we are now in the position to express the incremental instantaneous electromagnetic torque for the saturable SR machine as

$$
\begin{gathered}
T_{\theta \rightarrow \theta+1}=\left[\frac{\partial W^{\prime}}{\partial \theta}\right]_{i=\text { constant }} \approx \frac{\Delta W^{\prime}}{\Delta \theta}= \\
=\frac{1}{2 \Delta \theta}\left[\Delta L_{s}\left(i_{r}^{2}-i_{s} i_{r}\right)+\Delta L_{u} i_{s} i_{r}+\Delta \Psi_{s}\left(i_{r}-i_{s}\right)\right]
\end{gathered}
$$

Equation (5) is the fundamental expression for the incremental instantaneous electromagnetic torque production process taking place inside an SR machine operated either as a motor or a generator; be it a rotational or a translational device and, as is demonstrated in the examples below, magnetically saturating or non-saturating machines. The mathematical 
expression in (5) is strikingly simple compared to the previously discussed alternative approaches, for example as found in [4]-[6]. The following insights can be inferred from (5), namely that in order to maximize the incremental instantaneous electromagnetic torque production within an SR machine it is beneficial to maximize the differences between the respective inductances, the flux-linkages and the rated to saturating phase currents. This is most effectively achievable with an SR machine constructed from high quality electrical steel, having the smallest practically achievable aligned air gap length and allowing high peak currents in the phases.

\section{B. Examples of Static Torque Waveform Computations}

Having derived the fundamental expression for the instantaneous electromagnetic torque delivered by the generally saturable SR machine we can now use (5) to estimate the static torque waveforms for a typical SR machine optimized, built and tested under laboratory conditions - full details of the design parameters may be found in [13].

In order to effectively utilise expression (5) for the nonlinear flux-linkage map of Fig. 1, each of the respective fluxlinkage functions was linearized - as demonstrated in Fig. 2 using two straight line approximations. For simplicity, the resultant linearized inductances, associated linearized fluxlinkage axis intercepts and rated and saturated phase currents $\left(i_{r}, i_{s}\right)$ pertinent to Fig. 1 are summarised in Table I for each of the rotor angles from the fully unaligned to the fully aligned rotor positions for the chosen 18/12 topology as defined in (4).

TABLE I

THE LiNEARIZED INDUCTANCES, FLUX-LINKAGE AXIS INTERCEPTS AND PHASE CURRENT VALUES FOR THE INTEGRAL HORSEPOWER 18/12 SR MACHINE

\begin{tabular}{|c|c|c|c|c|c|c|}
\hline $\begin{array}{c}\theta \\
\left({ }^{\circ}\right)\end{array}$ & $L_{s \theta}(H)$ & $L_{u \theta}(H)$ & $\begin{array}{c}\Psi_{s}(@ \theta) \\
(V \cdot S)\end{array}$ & $i_{r}(A)$ & $i_{s}(A)$ & $i_{i}(A)$ \\
\hline 15 & 0.00124 & 0.00125 & -0.04254 & 300 & 50 & 0 \\
\hline 14 & 0.00125 & 0.00127 & -0.04292 & 300 & 50 & 100 \\
\hline 13 & 0.00130 & 0.00133 & -0.04348 & 300 & 50 & 200 \\
\hline 12 & 0.00136 & 0.00147 & -0.04157 & 300 & 50 & 300 \\
\hline 11 & 0.00139 & 0.00178 & -0.02758 & 300 & 50 & 300 \\
\hline 10 & 0.00136 & 0.00239 & 0.006449 & 300 & 50 & 300 \\
\hline 9 & 0.00131 & 0.00307 & 0.045494 & 300 & 50 & 300 \\
\hline 8 & 0.00125 & 0.00379 & 0.087888 & 300 & 50 & 300 \\
\hline 7 & 0.00118 & 0.00450 & 0.130288 & 300 & 50 & 300 \\
\hline 6 & 0.00110 & 0.00519 & 0.172839 & 300 & 50 & 300 \\
\hline 5 & 0.00100 & 0.00587 & 0.215771 & 300 & 50 & 300 \\
\hline 4 & 0.00090 & 0.00650 & 0.257885 & 300 & 50 & 300 \\
\hline 3 & 0.00080 & 0.00705 & 0.294194 & 300 & 50 & 80 \\
\hline 2 & 0.00074 & 0.00741 & 0.317508 & 300 & 50 & 35 \\
\hline 1 & 0.00071 & 0.00763 & 0.33219 & 300 & 50 & 18 \\
\hline 0 & 0.0007 & 0.00772 & 0.337859 & 300 & 50 & 0 \\
\hline
\end{tabular}

It should be noted at this point that for the fitting of the two straight lines to the fully unaligned flux-linkage curve at the rotor angle of $15^{\circ}$, as in Fig. 1, the two inductances in Table I are almost equal; however, as the rotor increments approach the fully aligned position at $0^{\circ}$ the two inductances become markedly different. Note, however, that the unaligned inductances and the associated flux-linkages do not feature in (5) and can well be left out of Table I, yet these are present in (2) for the maximum average torque computation.

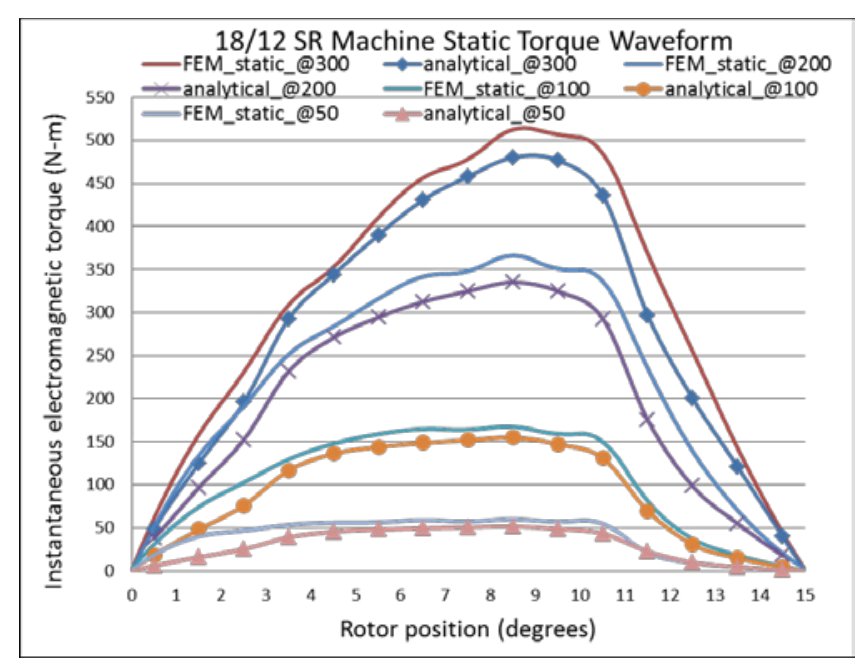

Fig. 4. Analytical and FEA generated static electromagnetic torque waveforms for the selected 18/12 SR machine.

Having the necessary data in Table I we can now use (5) to obtain the static electromagnetic torque waveform for the SR machine described in [13] for the range of rotor angles. For the 18/12 SR machine the range of the rotor angles is taken as half of the total rotor pole pitch angle since the periodicity of the flux-linkage map and of torque waveforms is repeated every half of the pole pitch, with the torque values alternating from the positive values to the negative, depending on the relative movement of the rotor pole to the respective stator pole while keeping the phase current constant throughout the complete rotor pitch angle. The resulting waveforms for the rated phase current are shown in Fig. 4, where they are also compared with the static torque waveforms generated using the open source magnetic field FEA package [7]. The analytical results in Fig. 4 do not fit the FEA results exactly; however, given that the values in Table I were obtained with linear approximations of the otherwise non-linear flux-linkage map without any curve fitting optimization techniques (except the manual graphical curve fitting method), the results in Fig. 4 may be considered to be in relatively very good agreement. Therefore, the results in Fig. 4 indicate that using (5) provides acceptably accurate estimates for the rated phase current static electromagnetic torque waveforms, in addition to not requiring any integration or non-physical coefficients in the process. The static electromagnetic torque results for some other phase currents that are below the rated value are also shown in Fig. 4, with any other such curve readily computable with (5).

\section{INTEGRAL HORSEPOWER SR MACHINE INSTANTANEOUS TORQUE ANALYSIS}

Having demonstrated the simplicity of obtaining the static instantaneous electromagnetic torque waveform for an integral horsepower 18/12 SR machine, we now proceed to estimate analytically the instantaneous electromagnetic torque under the time varying, as opposed to static, phase currents.

\section{A. Instantaneous Electromagnetic Torque Waveform Calculations}

We shall now use expression (5) to calculate instantaneous 
electromagnetic torque for the SR machine operated as a motor under time varying phase current waveforms. To this end expression (5) is modified as

$$
\begin{gathered}
T_{\theta \rightarrow \theta+1}=\left[\frac{\partial W^{\prime}}{\partial \theta}\right]_{i=\text { constant }} \approx \frac{\Delta W^{\prime}}{\Delta \theta}= \\
=\frac{1}{2 \Delta \theta}\left[\Delta L_{s}\left(i_{i}^{2}-i_{s} i_{i}\right)+\Delta L_{u} i_{s} i_{i}+\Delta \Psi_{s}\left(i_{i}-i_{s}\right)\right]
\end{gathered}
$$

where the instantaneous phase current $i_{i}$ varies incrementally from $\theta \rightarrow \theta+1$ as a function of the rotor position, and thus of time. The instantaneous flat-topped phase current values for each rotor position of the chosen SR machine are given in Table I.

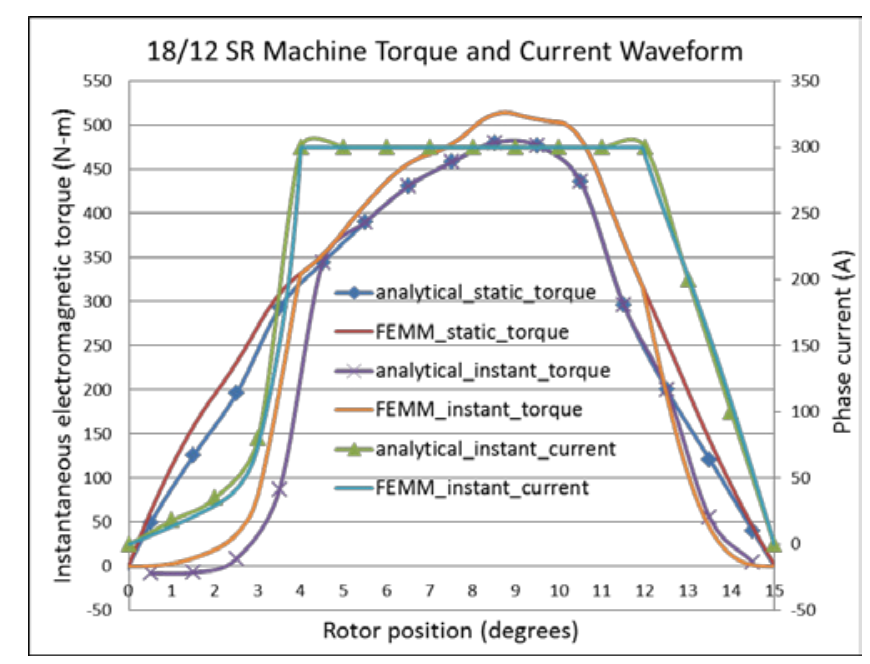

Fig. 5. Comparison of analytical and FEA generated instantaneous electromagnetic torque waveforms due to the instantaneous phase current waveform for the selected 18/12 SR machine at 500V DC bus voltage and 600rpm operating conditions.

The application of (6) in the motoring mode yields results shown in Fig. 5, where the phase current turn-on angle is at $15^{\circ}$ and the extinction angle is at $0^{\circ}$. In Fig. 5 the FEA based results were generated from the fully rigorous non-linear computer based simulation method as proposed in [14] in order to obtain the most reliable instantaneous flat-topped phase current waveform first, also given in numeric form in Table I, subsequently used in (6). Alternatively, the full derivation in reference [12] can be used to compute the instantaneous flat-topped phase current waveform analytically, if required, thus altogether avoiding computer based numerical analysis in the SR machine design process. The particular instantaneous phase current waveform in Fig. 5 resulted from the $500 \mathrm{~V}$ DC bus voltage at the rotor speed of 600rpm. The instantaneous torque waveform results obtained using (6) are of comparable accuracy to the computer based simulation results of the same SR machine accounting for the fully nonlinear flux-linkage map representation.

The instantaneous torque waveform results in Fig. 5 are plotted alongside the previously computed static torque waveforms of Fig. 4 in order to gain further physical insights. From Fig. 5 it can be deduced that in order to generate the maximum torque with the chosen SR machine design it is necessary to excite the motor phase as early as possible so as to build up the phase current to its peak value in the shortest possible time. Moreover, the current should be extinguished as late as possible, well past the fully aligned rotor position, since otherwise there is a potential for a significant wastage of torque if the current is extinguished too early, as seen in Fig. 5.

A further advantage of using expression (6) to estimate the instantaneous electromagnetic torque waveform due to the instantaneous current profiles is that the expression is valid for instantaneous phase current values $i_{i}$ considerably above and below the specified rated phase current value $i_{r}$. This variation of the current is taken automatically into account in (6), without the need to make any special adjustments due to the vector based co-energy definition in (2).

Further analysis of the instantaneous torque waveform as a function of the instantaneous phase current was conducted at the same operating conditions as in Fig. 5 but at the rotational speed of 1200rpm, with the results shown in Fig. 6.

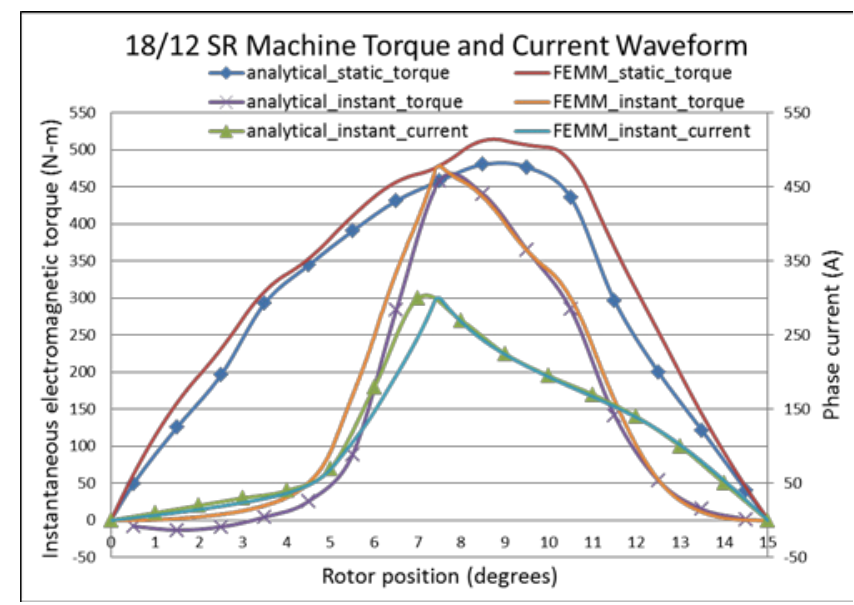

Fig. 6. Comparison of analytical and FEA generated instantaneous electromagnetic torque waveforms due to the instantaneous phase current values for the selected 18/12 SR machine design at 500V DC bus voltage and 1200 rpm operating conditions.

As in the case of Fig. 5, the results in Fig. 6 are also in very close agreement for the entire range of the instantaneous phase currents above and below the saturating phase current value. A similar comment regarding the maximum utilization of the available static torque for the given SR machine design - as was made in relation to Fig. 5 - applies to Fig. 6, where it may be seen that if the peak phase current value is not reached during the rotor advancing from the fully unaligned to the fully aligned position, the instantaneous torque is significantly lower compared to the static torque. This result implies the need to advance the excitation angle beyond the fully unaligned rotor position to capture as much of the static torque waveform as possible for the given dwell angle during which the phase current is conducted. Such physical insights into the machine operation are possible from the simple relationship in (6). 


\section{B. Maximum Instantaneous Electromagnetic Torque Generating Region}

Having performed extensive static and instantaneous torque waveform analysis with the simple yet relatively accurate expression (6), we proceed to conduct further analysis for the selected integral horsepower 18/12 SR machine to demonstrate the identification and characterization of the maximum instantaneous torque generating region.

The total amount of the potential co-energy $W$ ' for the given SR machine design can be found using (2) and this co-energy is represented by the total area bounded by the fully aligned and the fully unaligned flux-linkage curves as in Fig. 2. This co-energy area is the theoretical maximum that is never achieved in practice due to operational constraints of the maximum available DC bus voltage and phase current. Expression (4) can be used in a similar way to calculate the differential (or incremental) co-energy $d W^{\prime}$ as the rotor advances incrementally from the fully unaligned to the fully aligned position. In addition to the incremental co-energy, it is also possible - using (4) - to compute the cumulative coenergy for the given SR machine over the complete range of the rotor angles. These two results are shown in Fig. 7.

As can be seen from Fig. 7, the cumulative co-energy varies from zero to the maximum theoretical value for the given SR machine as the rotor advances from the fully unaligned to the fully aligned position. The cumulative co-energy curve displays the point of inflection at the rotor angle of approximately $9^{\circ}$, which coincides with the maximum value of the differential co-energy. Beyond the point of inflection, the differential co-energy starts to fall, indicating the diminishing value of the instantaneous torque. Thus, the results in Fig. 7 indicate that - similarly to the previous analysis using (6) - the maximum torque-generating region of the SR machine can be inferred and characterized. Specifically, driving the peak phase current while the rotor angle is at the fully unaligned rotor position has little effect on the torque generation value since the cumulative co-energy in this region is very small.

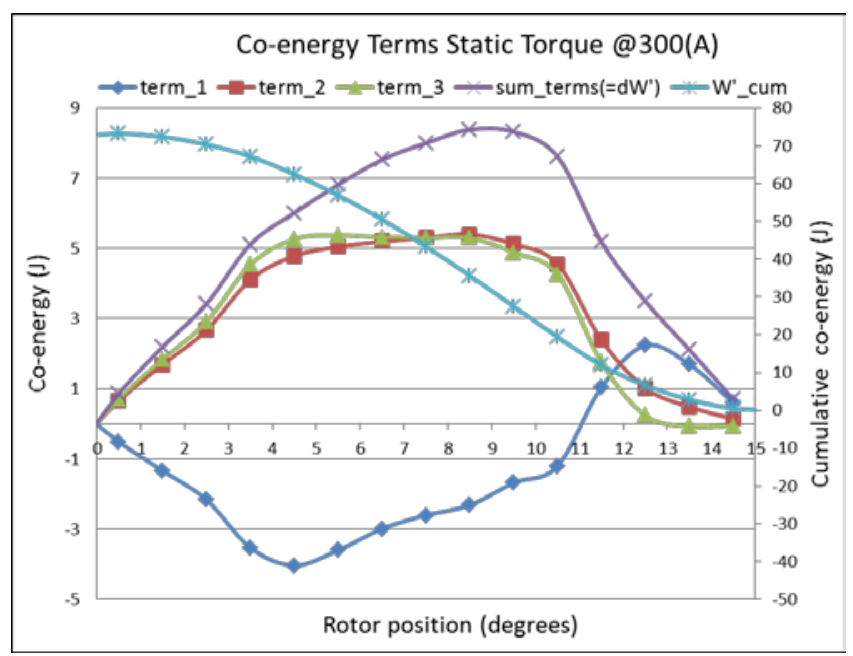

Fig. 7. The differential, the cumulative and the separate terms of co-energy of expression (4) as functions of the rotor position for the selected 18/12 SR machine.
However, as the rotor begins first to overlap and then fully align with the stator pole, the differential co-energy increases rapidly at first, subsequently levelling off just before the full alignment of the rotor pole. Similarly to the previous analysis using (6), the results in Fig. 7 can help to visualize the potential maximum-torque-per-ampere (MTPA) generating regions of the selected SR machine - this presenting itself as a trade-off between the highest torque and the most efficient machine operating modes.

Moreover, the three co-energy terms in (4) may also be separately visualised to reveal their unique interrelationship in the electromagnetic torque generation process inside the SR machine; these terms are plotted in Fig. 7. Remarkably, the second and the third co-energy terms in (4) are very similar for the entire range of the rotor angles and contribute positively to the total static torque, whereas the first term contributes positively and negatively the co-energy component depending on the respective rotor position. The uniqueness of this result as shown in Fig. 7 - cannot be underestimated; such insights can never be obtained with FEA based computations, herein revealing the relationship between the three co-energy components and their importance in the SR machine torque generating process. For example, the positive region of the first co-energy term in (4) in Fig. 7 shows clearly why it is advantageous to energize the phase coil early: if the current reaches the peak value before the rotor angle of $14^{\circ}$, a significant portion of the positive torque resulting from the first term is generated. Notwithstanding, it is recognized that all three co-energy terms in (4) have different multiplying phase current terms and if we plot the same relationships separately (for the previously shown in Fig 5 instantaneous phase current waveform) the following results emerge as shown in Fig. 8.

Comparing the results of Fig. 7 and Fig. 8, it can be seen that the instantaneous rated phase current value is reached at the point where the first co-energy term has a positive maximum and therefore the resulting positive torque is generated. As the rotor continues advancing towards the fully aligned position (zero degrees angle) the instantaneous phase current is being reduced in order to bring it to zero at the fully aligned position (and consequently the three co-energy terms), as in Fig. 7, and therefore their associated torque components are not fully realized. These, and similar, physical insights are very useful for the optimal control of the phase current waveforms and would not be possible to achieve with FEA simulations alone.

The separate co-energy terms in (4) were found for the static phase current waveform value $i_{r}$ - which is made to coincide with the saturation phase current $i_{s}$ of the chosen 18/12 SR machine as in Fig. 1 - giving the relationships as in Fig. 9. 


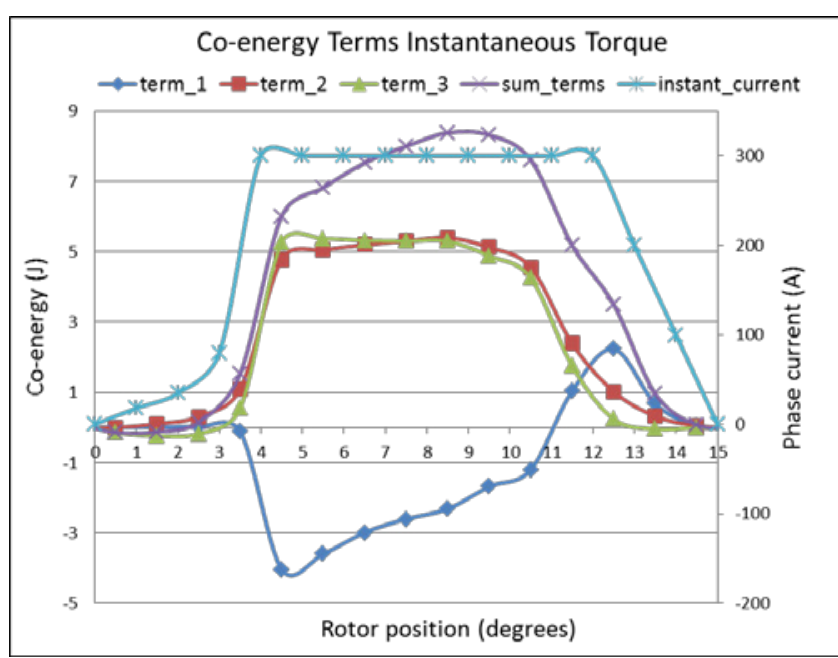

Fig. 8. The differential and the separate terms of co-energy of expression (4) as functions of the rotor position for the instantaneous phase current waveform of Fig. 5 for the selected 18/12 SR machine.

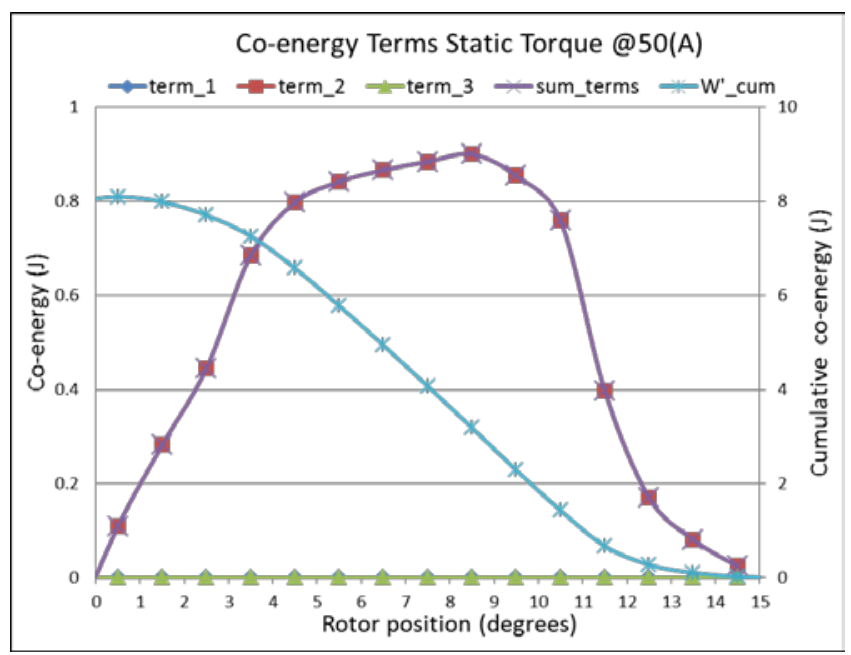

Fig. 9. The differential, the cumulative and the separate terms of co-energy of expression (4) as functions of the rotor position for the static torque waveform at 50A phase current for the selected 18/12 SR machine.

The plots in Fig. 9 were generated as for Fig. 7, but for the phase current significantly lower than the rated value and it comes as no surprise that the cumulative co-energy is reduced significantly. Further important point regarding the results in Fig. 9 is that only the second term of the co-energy in (4) remains effective under such low phase current values, thus equalling the differential co-energy, while the other two coenergy terms (term_1 and term_3) are reduced to zero. Clearly, this result should have been expected and confirms the validity and the generality of the proposed approach due to the classical instantaneous electromagnetic torque relationship for the non-saturated SR machine as [15]

$$
T_{e}=\frac{1}{2} i^{2} \frac{\partial L_{u}}{\partial \theta}
$$

Equation (7) should be compared with (5), after the rated phase current value $i_{r}$ is forced to be equal to the saturating phase current value $i_{s}$. In such case all but the second coenergy terms in (5) vanish and expression (5) reduces to (7), thus confirming the generality of the vector based approach presented in this work.

The final remark regarding the result of Fig. 9 is that if the generally saturable SR machine were to operate in the unsaturated flux-linkage region, under the less than saturating phase current values, the torque generating capability would be greatly reduced, as will be evident from the comparison of the cumulative co-energy in Fig. 7 and Fig. 9 and in accordance with the respective torque waveform of Fig. 4.

\section{FRACTIONAL HORSEPOWER SR MACHINE INSTANTANEOUS TORQUE ANALYSIS}

Having demonstrated the computational convenience, insightfulness and accuracy of expressions (5) and (6) for the integral horsepower SR machine, we now proceed to apply the analysis to a selected fractional horsepower SR machine designed for an electric bicycle application. The main purpose for the analysis of the fractional horsepower SR machine is to demonstrate that the proposed approach works equally well for relatively high power and the relatively low power machines. The performance ratings and the design parameters of the selected 18/12 SR machine are summarized in Table II [16].

TABLE II

Fractional HoRSEPOWER 18/12 THREE PHASE SR MACHINE DESIGN

\begin{tabular}{c|c}
\multicolumn{2}{c}{ PARAMETERS } \\
\hline Design Parameters & SRM/G \\
\hline Outer diameter and stack length $(\mathrm{mm})$ & 100 and 80 \\
\hline Air gap length $(\mathrm{mm})$ & 0.3 \\
\hline Iron core material & M19-0.35 (mm) \\
\hline Coil wire diameter (mm) & 1.0 \\
\hline Number of turns & 16 \\
\hline Slot fill factor $(\%)$ & 57 \\
\hline Current, peak (A) $(\mathrm{m})$ & 30 \\
\hline DC-bus voltage $(\mathrm{V})$ & 45 \\
\hline RMS current (at 1200rpm) $(\mathrm{A})$ & 20 \\
\hline Max Torque (at 1200rpm) $(\mathrm{Nm})$ & 3 \\
\hline Max Torque (at 600rpm) $(\mathrm{Nm})$ & 6.0 \\
\hline
\end{tabular}

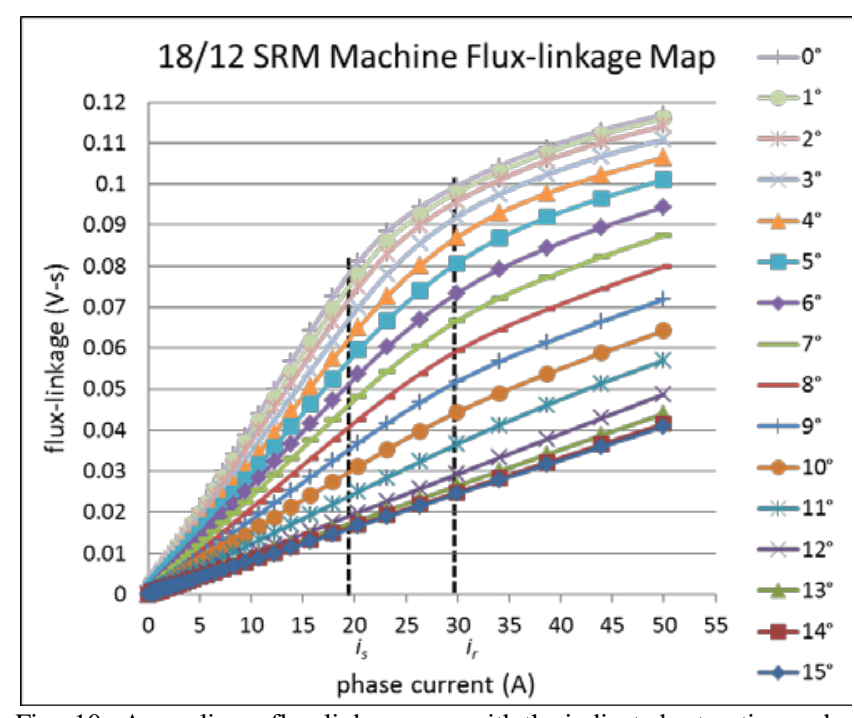

Fig. 10. A non-linear flux-linkage map with the indicated saturation and rated phase currents for the fractional horsepower 18/12 SR machine designed for electric bicycle application. 
As in the case of the integral horsepower SR machine analysis, for the fractional horsepower SR machine the nonlinear flux-linkage map is obtained first - as shown in Fig. 10 - prior to the linearization. Once the non-linear flux-linkage map is available, the linear curve fitting is applied with the resulting linearized inductances, associated flux-linkage axis intercepts and rated and saturating phase currents pertinent to Fig. 10, as detailed in Table III.

\section{TABLE III}

THE LINEARIZED INDUCTANCES, FLUX-LINKAGE AXIS INTERCEPTS AND PHASE CURRENT VALUES FOR THE FRACTIONAL HORSEPOWER 18/12 SR

\begin{tabular}{|c|c|c|c|c|c|c|}
\hline & & $\Psi_{s}(@ \theta)$ \\
$\theta(V)$ & $L_{s \theta}(H)$ & $L_{u \theta}(H)$ & $i_{r}(A)$ & $i_{s}(A)$ & $i_{i}(A)$ \\
\hline 15 & 0.00082 & 0.00082 & 0.04085 & 30 & 20 & 0 \\
\hline 14 & 0.00083 & 0.00083 & 0.04157 & 30 & 20 & 14.2 \\
\hline 13 & 0.00088 & 0.00088 & 0.04407 & 30 & 20 & 24.2 \\
\hline 12 & 0.00097 & 0.00098 & 0.04878 & 30 & 20 & 29.2 \\
\hline 11 & 0.00108 & 0.00123 & 0.05704 & 30 & 20 & 30 \\
\hline 10 & 0.00112 & 0.00153 & 0.06424 & 30 & 20 & 30 \\
\hline 9 & 0.00118 & 0.00181 & 0.07190 & 30 & 20 & 30 \\
\hline 8 & 0.00121 & 0.00209 & 0.07986 & 30 & 20 & 30 \\
\hline 7 & 0.00126 & 0.00237 & 0.08748 & 30 & 20 & 30 \\
\hline 6 & 0.00128 & 0.00263 & 0.09441 & 30 & 20 & 26.6 \\
\hline 5 & 0.00132 & 0.00292 & 0.10106 & 30 & 20 & 19.7 \\
\hline 4 & 0.00133 & 0.00319 & 0.10647 & 30 & 20 & 14.7 \\
\hline 3 & 0.00138 & 0.00343 & 0.11090 & 30 & 20 & 10.5 \\
\hline 2 & 0.00138 & 0.00367 & 0.11424 & 30 & 20 & 6.7 \\
\hline 1 & 0.00140 & 0.00383 & 0.11607 & 30 & 20 & 3.2 \\
\hline 0 & 0.00140 & 0.00398 & 0.11697 & 30 & 20 & 0 \\
\hline
\end{tabular}

The values in Table III are subsequently used in expressions (4) - (6) for the static and instantaneous torque calculations.

\section{A. Instantaneous Electromagnetic Torque Waveform Calculations}

The instantaneous torque waveform calculation is performed using (5), with the resulting calculated torque compared against the torque waveform in Fig. 11 obtained using fully non-linear computer based numerical computations.

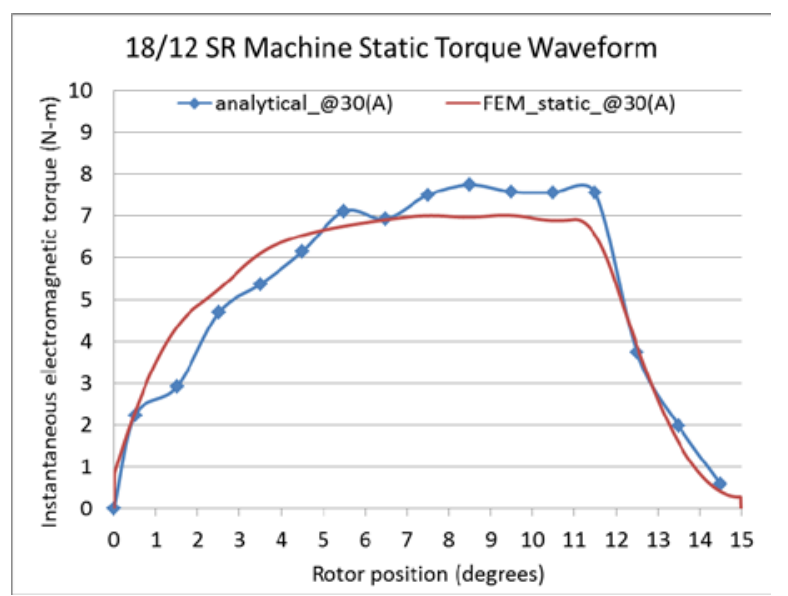

Fig. 11. Analytical and FEA generated static instantaneous electromagnetic torque waveforms comparison for the selected fractional horsepower 18/12 SR machine design.

From Fig. 11 it can be seen that the analytical electromagnetic torque waveform is less precise than for the integral horsepower SR machine as in Fig. 4. This discrepancy is likely to be due to the less sharply pronounced saturation point of the flux-linkage map in Fig. 10 for the fractional horsepower SR machine making it more difficult to select the single value of the saturating phase current $i_{s}$ which would ensure best accuracy of the torque waveform.

Using expression (6), the analytical instantaneous torque waveforms are obtained for the instantaneous phase current values as detailed in Table III and shown in Fig. 12, also comparing the instantaneous torque waveforms obtained with the non-linear computer based numerical simulations for the operating speed of 500rpm and the DC bus voltage of $45 \mathrm{~V}$.

As evident from Figs. 11 and 12, the analytically produced torque waveforms are of sufficient accuracy for the purpose of the initial electromagnetic design of the SR machine.

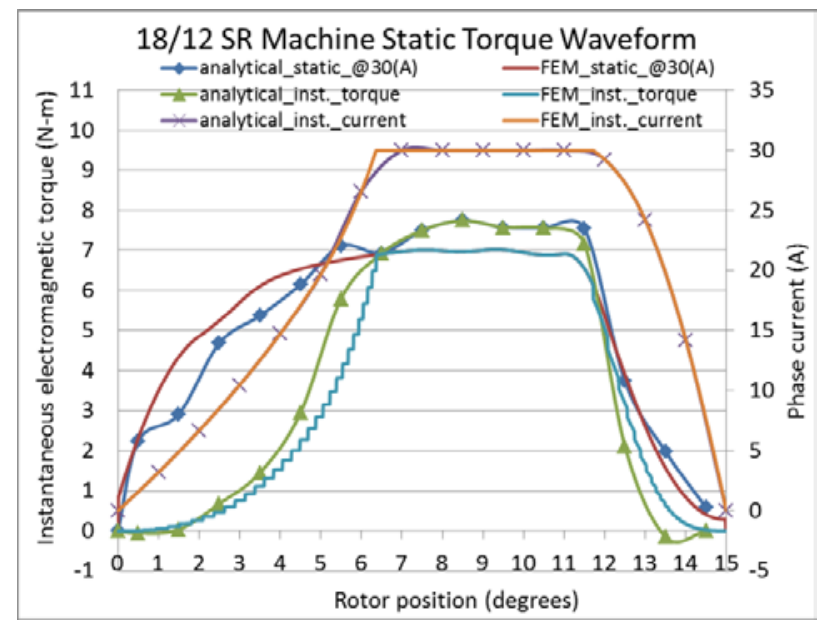

Fig. 12. Comparison of analytical and FEA generated instantaneous electromagnetic torque waveforms due to the instantaneous phase current waveform for the selected 18/12 SR machine design at 45V DC bus voltage and 500rpm operating conditions.

\section{B. Maximum Instantaneous Electromagnetic Torque Generating Region}

The maximum instantaneous torque-generating region is found using (4) with each of the co-energy terms represented separately and as a sum of terms as shown in Fig. 13.

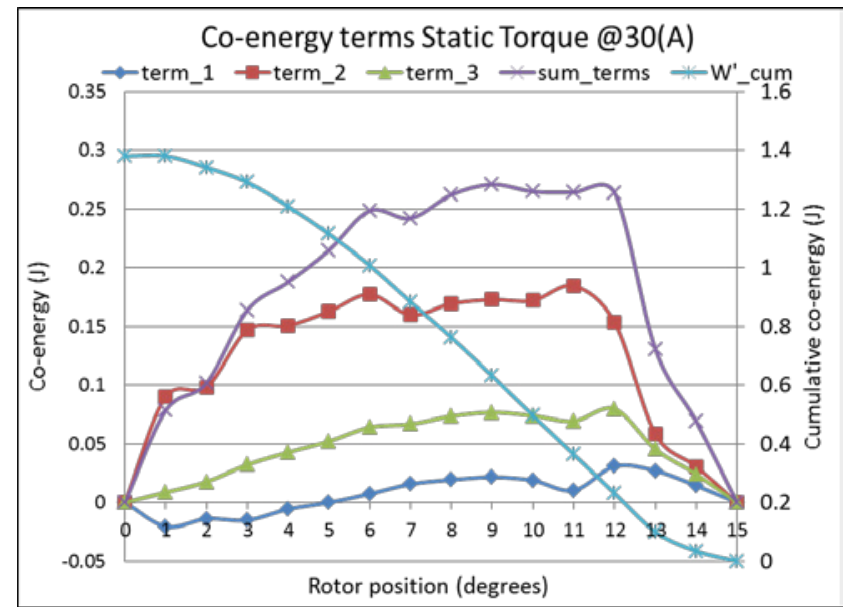

Fig. 13. The differential, the cumulative and the separate terms of co-energy of expression (4) as functions of the rotor position for the selected fractional horsepower 18/12 SR machine. 
In Fig. 13, similarly to Fig. 7, the separately plotted coenergy terms reveal that the negative part of the first term in (4) is delayed up to the point of the rotor position of $4^{\circ}$ and compared to the result of Fig. 7 - is much shorter than for the same topology 18/12 SR machine. This suggests that machine topology does not on-its-own define the negative part of the first co-energy term and thus the specific geometric parameters are a probable cause of such phenomenon.

\section{Computational AsPects of the Estimation Of the INSTANTANEOUS ELECTROMAGNETIC TORQUE WAVEFORMS}

The methodology put forward here for the computation of the instantaneous electromagnetic torque waveform of saturated SR machines is a comparatively simple analytical procedure for what is essentially a non-linear electromagnetic problem. The proposed approach, expressions (5) and (6) in particular, could be further refined based on vector analysis in order to improve the accuracy even further; however, providing this does not lead to overly complicated expressions that are difficult to interpret and thus convey no physical meaning. In this section, we present a number of suggestions as to the directions of the refinements of the proposed approach that could lead to the results accuracy improvements using (5) and (6).

\section{A. 3D and 2D Electromagnetic Analysis Flux-linkage Maps}

As SR machines are built as 3D objects, their analysis should ideally be performed using 3D computational techniques. Such 3D analysis is normally a complex and lengthy task but, if performed competently, will result in the most accurate performance predictions. Therefore, the nonlinear flux-linkage maps, as in Figs. 1 and 10, could be argued to require $3 \mathrm{D}$ analysis to provide the most accurate linearized curve fitting prior to the use of expressions (5) and (6) for the torque calculations. However, the differences between 3D and 2D flux-linkage maps are not of immediate concern when using the approach based on (5) as the proposed methodology focuses on the preliminary stages of the design where 3D FEA is usually not warranted. It is nevertheless important to note that a more accurate non-linear flux-linkage representation, such as obtained with 3D electromagnetic modelling, will result in increased accuracy of the linearized curve fitting and therefore more accurate instantaneous torque calculations. Therefore, whenever possible and practical, the 3D based electromagnetic analysis generated flux-linkage maps are recommended.

\section{B. Co-energy Central-, Forward-, and Backward-difference Schemes}

As may be seen from the expressions (1) and (4) - and the calculated cumulative co-energy as a function of the rotor position, as in Figs. 7 and 13 - the resulting incremental torque values may be assumed to occur between the two consecutive rotor positions of interest, as shown schematically in Fig. 14.

It follows from Fig. 14 that the instantaneous torque value $T$ may be found as a ratio of the cumulative co-energy difference to the rotor position difference - which is in fact the gradient of the co-energy curve at that point. A question arises, however, where to assign this calculated instantaneous torque value. Given that the rotor position is advanced with a fixed value increment, the calculated torque value may be allocated to the starting rotor position, the incremented rotor position or at the centre between these two rotor positions. These three positions will be referred to as a calculation of the torque with expression (5) using forward-, backward-, or the centraldifference calculation schemes, respectively.

The implication of these three possible schemes is that the representation of the calculated torque values with respect to the rotor position, for example as plotted in Figs. 5 and 6, have to be considered. That is, the assigned calculated torque values should correspond to the rotor positions, in addition to the phase current values, especially if the instantaneous torque values are being calculated. Moreover, expression (1) is approximated with (5) with the assumption of the constant current condition for the co-energy increment of interest. Consequently, a proper assignment of the calculated torque with respect to the rotor position and the instantaneous current value will result in the most accurate representation of the torque waveform.

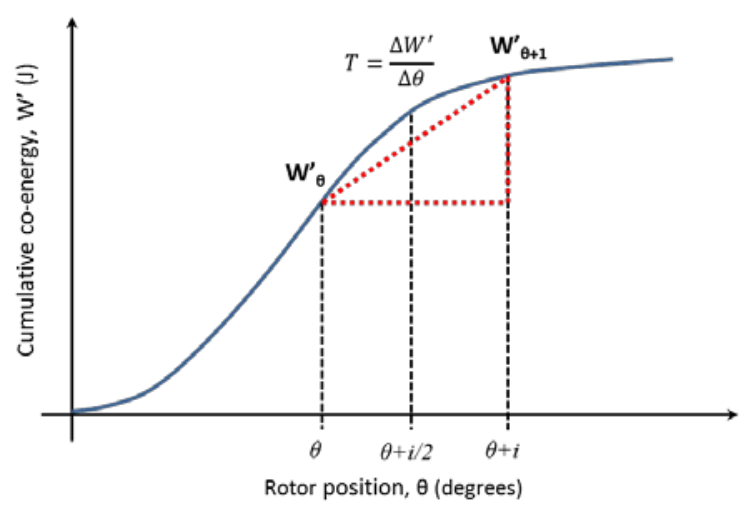

Fig. 14. The cumulative co-energy curve as a function of the rotor position and the calculation of the resulting torque using the central difference scheme.

A further important point with regard to Fig. 14 is that the calculated torque value will be directly affected by the choice of the rotor position increment $i$. For example, if the magnitude of the increment $i$ is selected as too large, the resulting co-energy gradient value - and therefore the torque is likely to be much less accurate compared to a very small magnitude of the increment. However, since the generated non-linear flux-linkage map is in the form of the predetermined discrete rotor position increments, the increment $i$ is fixed from the outset, unless further interpolation between the generated non-linear flux-linkage curves is performed with respect to the rotor position. Such further computational effort, before the flux-linkage map is linearized via curve fitting, is best avoided through careful consideration of the analysed SR machine topology in terms of the number of the rotor and stator poles. For example, if for a given SR machine the number of the rotor poles is large then 
the required rotor angle increment will be relatively small and the non-linear flux-linkage map should be generated accordingly in order to yield accurate instantaneous torque calculations using (5).

\section{Accurate Linear Representation of the Non-linear Flux- linkage Map}

One of the supporting arguments in favour of the proposed linearization of the non-linear flux-linkage map of the saturable SR machine, as in Fig. 2, is good accuracy of the calculated instantaneous torque despite application of a relatively simple curve fitting technique. The resulting torque expression offers simplicity and is intuitive due to a small number of terms in (5); it is therefore much preferred to the alternative expressions (mentioned in the Introduction) which may contain a large number of non-linear curve fitting coefficients. However, a further refinement of the proposed analytical torque calculation is possible by applying a larger number of linear curve fitting procedures to all of the fluxlinkage curves; an example for a particular curve is shown in Fig. 15, where only the fully aligned flux-linkage curve is shown, while in practice all of the curves could be treated in a similar manner.

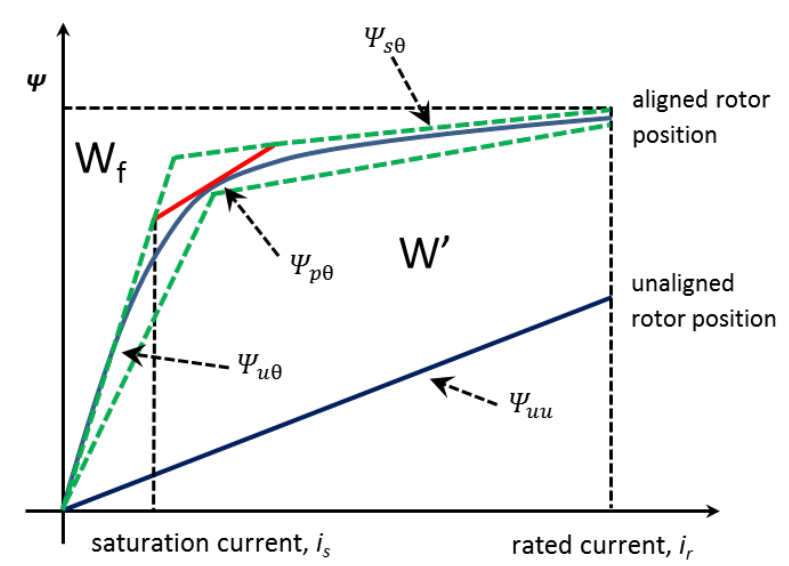

Fig. 15. Accurate representation of the non-linear flux-linkage curve using three linear curve fits.

Referring to Fig. 15, given that the unsaturated part of the fully aligned flux-linkage curve is geometrically relatively well defined, it can be linearized using a straight line $\Psi_{u \theta}$. In a similar way, the fully saturated part of the fully aligned fluxlinkage curve is also relatively well defined and therefore can be represented by $\Psi_{s \theta}$. However, these two linear curve fittings for the highly non-linear curve may sometimes in practice result in an overestimation or underestimation of the co-energy $W^{\prime}$, as it is proportional to the area bound by these two curves and the fully unaligned unsaturated flux-linkage curve $\Psi_{u u}$. The simplest way to prevent this unwelcome inaccuracy is to use a third linear curve fit to represent the small - although not easy to specify - partially saturated part of the flux-linkage curve defined as $\Psi_{p \theta}$. Therefore, referring to the graphical representation in Fig. 15, it may be argued that taking into account the proposed third linear curve fitting will improve the accuracy of the co-energy $W$ ' representation and therefore the instantaneous torque calculation. Inevitably this modification would result in the final expression becoming more complicated and thus less intuitive; it is therefore suggested that the additional term should be used as a last resort only if the flux-linkage map is very difficult to approximate using only two linearized curve technique.

\section{DISCUSSION}

The proposed analytical computational procedure for the estimation of the instantaneous electromagnetic torque waveforms of saturable SR machines is both simple and insightful, as evident from (5) and (6) as well as the presented numerical results. This is very encouraging, since the suggested method can be used to explain the saturable SR machine torque generating process intuitively and in physically meaningful terms, especially to those not familiar with this particular electric machine technology.

The advantages of the proposed methodology in comparison with the reviewed in Section I existing alternative analytical approaches, for example [4]-[6], may be summarized as follows. The most important benefit arising from the presented vector based instantaneous torque analysis, and the resulting equations (5) and (6), is that these expressions are remarkably simple to interpret as they feature quantities that are physically meaningful. The mentioned alternative analytical approaches were seen to lack this physical meaningfulness and insightfulness due to the use of the optimized non-linear curve fitting coefficients. A further important advantage of the proposed approach is that it avoids direct integration and for this reason is simpler to use for the analysis of the SR machines compared to the methods that necessitate analytical integration with the resulting coefficients offering no physical meaning. The final advantage of the proposed scheme is that the calculation is based on successive co-energy differences instead of geometric series based summation expressions that do not convey any physical representation of the electromagnetic processes taking place inside the machine. Therefore, the proposed analytical approach is claimed to be highly suitable for the description and characterization of the electromagnetic energy conversion processes in the SR machines.

As is the case with any analytical expressions for the solution of non-linear magnetic problems, the proposed vector based analysis has a particular disadvantage in that the vectorbased method is linear in nature, while it is aimed at a nonlinear problem. Therefore, the numerical solution accuracy offered with the proposed linear vector based method can never approach the accuracy of non-linear computer based computational methods. Notwithstanding, the error of the answer resulting from the proposed vector based analysis can be reduced, especially in the analysis of SR machines constructed from high performance electromagnetic steels that exhibit high saturation flux densities and thus give rise to the very sharp and hence well-defined saturation knee-point of the non-linear flux-linkage curve. Therefore, the linear vector based modelling of the flux-linkage curves with highly pronounced saturation point will result in very small 
numerical discrepancies against fully non-linear computer based simulations.

Finally, it should be pointed out that the proposed approach exploiting vector analysis is potentially very general in scope as many other electromagnetic devices can be analysed in terms of flux-linkage maps. It may be argued that expressions (5) and (6), in particular, are highly relevant and offer suitable analytical formulations to other electromechanical apparatus operating on the principle of an air-gapped electromagnet, including such devices as tractive electromagnets, contactors, power relays and similar. Therefore, the theory presented above can be very beneficial as a subsidiary analysis procedure, especially in the preliminary stages of the design, helping engineers to estimate rapidly and with good physical insight the potential energy conversion capability of the device before proceeding to the more advanced electromagnetic design optimization procedures [8].

\section{CONCLUSION}

An analytically simple and numerically relatively accurate computational procedure for the estimation of the instantaneous electromagnetic torque waveforms for saturable SR machines has been presented. The proposed analytical approach relies on vector representation of the flux-linkage map and offers helpful physical insight into the electromagnetic energy conversion processes in the machines operating under deep magnetic saturation as well as in the unsaturated regime. The proposed approach based on the vector analysis of the flux-linkage maps shows good agreement with the computer generated FEA results. Furthermore, it is argued that the presented methodology will be a suitable magnetic analysis procedure for other electromechanical devices, such as contactors, tractive electromagnets and power relays, offering rapid design.

\section{ACKNOWLEDGMENT}

This work was supported in part by the UK Engineering and Physical Sciences Research Council (EPSRC) under Grant EP/P010164/1.

\section{REFERENCES}

[1] T. J. E. Miller, Switched reluctance motors and their control. Hillsboro, OH : Oxford: Magna Physics Pub. ; Clarendon Press, 1993.

[2] R. Krishnan, Switched reluctance motor drives: modeling, simulation, analysis, design, and applications. Boca Raton, FL: CRC Press, 2001.

[3] M. R. Harris and J. K. Sykulski, 'Simple method for calculating the peak torque of a switched reluctance motor: a computational investigation', COMPEL - Int. J. Comput. Math. Electr. Electron. Eng., vol. 11, no. 1, pp. 193-196, Jan. 1992, doi: 10.1108/eb051785.

[4] T. J. E. Miller and M. McGilp, 'Nonlinear theory of the switched reluctance motor for rapid computer-aided design', IEE Proc. B Electr. Power Appl., vol. 137, no. 6, p. 337, 1990, doi: 10.1049/ipb.1990.0042.

[5] D. A. Andrade and R. Krishnan, 'Characterization of switched reluctance machines using Fourier series approach', in Conference Record of the 2001 IEEE Industry Applications Conference. 36th IAS Annual Meeting (Cat. No.01CH37248), Chicago, IL, USA, 2001, vol. 1, pp. 48-54, doi: 10.1109/IAS.2001.955391.

[6] C. Vaithilingam, N. Misron, M. Zare, I. Aris, and M. Marhaban, 'Computation of Electromagnetic Torque in a Double Rotor Switched
Reluctance Motor Using Flux Tube Methods', Energies, vol. 5, no. 12, pp. 4008-4026, Oct. 2012, doi: 10.3390/en5104008.

[7] D. Meeker, Finite Element Method Magnetics (FEMM), FEMM Reference Manual, http://www.femm.info/wiki/Documentation/ , accessed on 5 September 2017.

[8] A. Stuikys and J. Sykulski, 'Rapid Multi-Objective Design Optimization of Switched Reluctance Motors Exploiting Magnetic Flux Tubes', IET Sci. Meas. Technol., Nov. 2017, doi: 10.1049/ietsmt.2017.0213.

[9] A. Radun, 'Analytical calculation of the switched reluctance motor's unaligned inductance', IEEE Trans. Magn., vol. 35, no. 6, pp. 44734481, Nov. 1999, doi: 10.1109/20.809140.

[10] A. Radun, 'Analytically computing the flux linked by a switched reluctance motor phase when the stator and rotor poles overlap', IEEE Trans. Magn., vol. 36, no. 4, pp. 1996-2003, Jul. 2000, doi: $10.1109 / 20.875277$.

[11] H. S. M. Coxeter and S. L. Greitzer, Geometry revisited, 10. print. Washington: Mathematical Assoc. of America, 2005.

[12] A. Stuikys and J. K. Sykulski, 'Average Rated Torque Calculations for Switched Reluctance Machines Based on Vector Analysis' in Modelling and Control of Switched Reluctance Machines, Rui Esteves Araújo, José Camacho, Eds.. InTechOpen, 2020.

[13] M. Takeno, A. Chiba, N. Hoshi, S. Ogasawara, M. Takemoto, and M. A. Rahman, 'Test Results and Torque Improvement of the $50-\mathrm{kW}$ Switched Reluctance Motor Designed for Hybrid Electric Vehicles', IEEE Trans. Ind. Appl., vol. 48, no. 4, pp. 1327-1334, Jul. 2012, doi: 10.1109/TIA.2012.2199952.

[14] J. M. Stephenson and J. Čorda, 'Computation of torque and current in doubly salient reluctance motors from nonlinear magnetisation data', Proc. Inst. Electr. Eng., vol. 126, no. 5, p. 393, 1979, doi: 10.1049/piee.1979.0095.

[15] S. A. Nasar, 'D.C.-switched reluctance motor', Proc. Inst. Electr. Eng., vol. 116, no. 6, p. 1048, 1969, doi: 10.1049/piee.1969.0194.

[16] Komolafe, Wagih, Valavan, Ahmed, Stuikys, and Zaghari, 'A Smart Cycling Platform for Textile-Based Sensing and Wireless Power Transfer in Smart Cities', Proc. MDPI, vol. 32, no. 1, p. 7, Dec. 2019, doi: 10.3390/proceedings2019032007.

Aleksas Stuikys received the B.Sc. degree in Mechanical Engineering and M.Sc. degree in Advanced Engineering Design from Oxford Brookes University, Oxford, UK, in 2009 and 2011 respectively. He obtained the doctoral degree from the Institute for Complex Systems Simulation, School of Electronics and Computer Science, University of Southampton, Southampton, UK in 2018.

Dr. Stuikys is founder of RETORQ Motors Ltd. - company specializing in the advanced electric traction motor and propulsion systems technology research and development for the electrification of transportation. His research interests span the fields of the propulsion systems, including electric traction motors; their modelling, simulation and design for the electric and hybrid vehicles and for the sustainable transport in general. The research interests also include the modelling, design and control aspects of switched reluctance machines based traction systems.

Bahareh Zaghari received M.Sc degree (1st class honors) in Advanced Mechanical Engineering (Mechatronics) in 2012 from the University of Southampton, UK. She received her Ph.D. in Dynamic analysis of a nonlinear parametrically excited system using electromagnets from Institute of Sound and Vibration (ISVR) at the University of Southampton in 2017. She was a Research Fellow at the school of Electronics and Computer Science, and she has been working on the design of smart systems, such as the next generation of jet engines and smart cities. She is currently a Lecturer in Propulsion Integration at Cranfield University and a visiting fellow at University of Southampton. Dr. Zaghari has received several awards for her outstanding performance in supporting women in academia.

Jan Sykulski (M'92-SM'93-F'2010) is Professor of Applied Electromagnetics at the University of Southampton, UK. His personal research is in development of fundamental methods of computational electromagnetics, power applications of high temperature superconductivity, simulation of coupled field systems and design and optimization of electromechanical devices. He has published over 430 scientific papers and co-authored four books. He is founding Secretary of International Compumag Society, Visiting Professor at universities in Canada, France, Italy, Poland and China, Editor of IEEE Transactions on Magnetics, Editor-in-chief of IET SMT, Editor-in-chief of COMPEL (Emerald) and member of International 
Steering Committees of several international conferences. He is Fellow of IEEE (USA), Fellow of the Institution of Engineering and Technology (IET), Fellow of the Institute of Physics (IoP), Fellow of the British Computer Society (BCS), Doctor Honoris Causa of Universite d'Artois, France, and has an honorary title of Professor awarded by the President of Poland. 